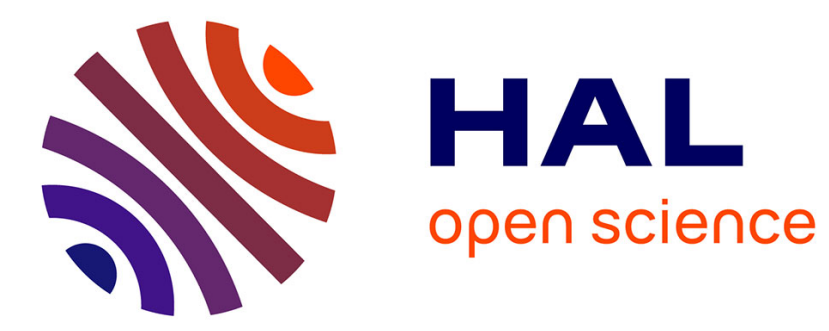

\title{
Implicit frictional-contact model for soft particle systems
}

Saeid Nezamabadi, Farhang Radjai, Julien Averseng, Jean-Yves Delenne

\section{To cite this version:}

Saeid Nezamabadi, Farhang Radjai, Julien Averseng, Jean-Yves Delenne. Implicit frictional-contact model for soft particle systems. Journal of the Mechanics and Physics of Solids, 2015, 83, pp.72-87. 10.1016/j.jmps.2015.06.007 . hal-01223541

\section{HAL Id: hal-01223541 \\ https://hal.science/hal-01223541}

Submitted on 17 Mar 2016

HAL is a multi-disciplinary open access archive for the deposit and dissemination of scientific research documents, whether they are published or not. The documents may come from teaching and research institutions in France or abroad, or from public or private research centers.
L'archive ouverte pluridisciplinaire HAL, est destinée au dépôt et à la diffusion de documents scientifiques de niveau recherche, publiés ou non, émanant des établissements d'enseignement et de recherche français ou étrangers, des laboratoires publics ou privés. 


\title{
Implicit frictional-contact model for soft particle systems
}

\author{
Saeid Nezamabadi ${ }^{a, b, *}$, Farhang Radjai ${ }^{a, c}$, Julien Averseng ${ }^{a}$, Jean-Yves Delenne ${ }^{b}$ \\ a LMGC, UMR 5508 CNRS - Université de Montpellier, 163 rue Auguste Broussonnet, 34090 Montpellier, France \\ ${ }^{\mathrm{b}}$ IATE, UMR1208 INRA - CIRAD - Université de Montpellier - SupAgro, 34060 Montpellier, France \\ c $<M S E>{ }^{2}$, UMI 3466 CNRS-MIT, CEE, Massachusetts Institute of Technology, 77 Massachusetts Avenue, Cambridge 02139, USA
}

\begin{abstract}
A B S T R A C T
We introduce a novel numerical approach for the simulation of soft particles interacting via frictional contacts. This approach is based on an implicit formulation of the Material Point Method, allowing for large particle deformations, combined with the Contact Dy-namics method for the treatment of unilateral frictional contacts between particles. This approach is both precise due to the treatment of contacts with no regularization and artificial damping parameters, and robust due to implicit time integration of both bulk degrees of freedom and relative contact velocities at the nodes representing the contact points. By construction, our algorithm is capable of handling arbitrary particle shapes and deformations. We illustrate this approach by two simple 2D examples: a Hertz contact and a rolling particle on an inclined plane. We also investigate the compaction of a packing of circular particles up to a solid fraction well above the jamming limit of hard particles. We find that, for the same level of deformation, the solid fraction in a packing of frictional particles is above that of a packing of frictionless particles as a result of larger particle shape change.
\end{abstract}

Keywords:

Material point method; Contact dynamics; Granular materials; Soft-particle systems

\section{Introduction}

Granular materials are of crucial importance in industrial transformation processes dealing with powders and grains as well as in geological flows and applications involving geomaterials (soils, rocks and concrete) (Nedderman, 1992; Jaeger and Nagel, 1996; Turner and Schuster, 1996). These materials present a complex pressure-dependent, density-dependent and rate-dependent mechanical behavior, which has attracted extensive modeling effort in different communities (Wood, 1990; Nedderman, 1992; Radjai et al., 2004; GDR-MiDi, 2004). Granular materials present also a broad range of characteristics related to the shapes and size distributions of their constitutive particles (Herrmann et al., 2003; Donev et al., 2004; Antony and Kuhn, 2004; Voivret et al., 2007; Azéma et al., 2009; Azéma and Radjai, 2010; CEGEO et al., 2012). Most recent fundamental research has focused on the scale-up of mechanical properties from the scale of particles and their interactions, characterized by a disordered microstructure and highly inhomogeneous stress transmission (Radjai et al., 1998; Kruyt, 2003; Majmudar and Behringer, 2005; Agnolin and Roux, 2007a; Richefeu and El Youssoufi, 2009).

Granular materials have been mainly modeled as a collection of undeformable particles. The elastic deformations are assumed to be concentrated at the contact points, and thus described as a function of the rigid-body degrees of freedom of the particles (Cundall and Strack, 1979; Matuttis et al., 2000; Radjai and Richefeu, 2009). This hard-particle approximation is

\footnotetext{
* Corresponding author at: LMGC, UMR 5508 CNRS - Université de Montpellier, 163 rue Auguste Broussonnet, 34090 Montpellier, France.

E-mail address: saeid.nezamabadi@umontpellier.fr (S. Nezamabadi).
} 
the physical ground of the popular Discrete Element Method (DEM) for the simulation of granular materials composed of model particles interacting via frictional contacts (Cundall and Strack, 1979). The numerical strategies based on DEM have the advantage of being robust. They include (1) Molecular Dynamics (MD), with an explicit time-stepping scheme based on force laws with strain variables derived from rigid-particle degrees of freedom (Cundall and Strack, 1979; Matuttis et al., 2000), and (2) Contact Dynamics (CD), which employs an implicit-type time integration scheme based on contact laws expressing the mutual exclusions of the particles and their Coulomb-like frictional behavior (Moreau, 1994; Jean, 1998; Radjai and Dubois, 2011).

The material behavior of the particles may be accounted to some extent in DEM through the force laws. In particular, the Hertz law and its generalization to frictional particles involve the elastic moduli and coefficient of friction of the particles Agnolin and Roux (2007a,b). Moreover, to first order in contact deflection $\delta$, the latter may be replaced by the overlap between two undeformable spherical particles (Leroy, 1985). This approximation holds as far as the average stress $p$ is small compared to Young's modulus $E$ of the particles. In many applications, however, this approximation is too crude, and the particles may undergo large elastic or inelastic deformations. For example, metallic powders are mostly composed of soft particles that may deform plastically without rupture (Rabinowicz, 1965). In the same way, many products in pharmaceutical and food industries are soft-particle materials. This broad class of materials may be further extended to colloidal pastes, vesicles, microgels and many suspensions if particle sizes below $1 \mu \mathrm{m}$ are considered (Cruz et al., 2002; Cloitre et al., 2003; Bonnecaze and Cloitre, 2010). All such materials may undergo volume change as a consequence of particle rearrangements, as in hard-particle materials. But what makes them different is their property of volume change by particle shape and size change under moderate external loads. This leads to enhanced space filling compared to hard particles in which the packing fraction cannot exceed the random close packing (RCP) limit (Berryman, 1986; Torquato et al., 2000). The compaction, shear behavior and other rheological properties of soft-particle systems beyond this "jamming" limit have remained largely unexplored.

For realistic modeling of soft-particle materials at large deformations, it is necessary to combine a continuum representation of the particles, allowing for their deformation according to a prescribed constitutive model, with appropriate frictional contact conditions between particles. In this regard, a promising framework is provided by meshless models that have already been applied to problems of solid mechanics involving large deformations. One of these numerical models is now mostly known as Material Point Method (MPM) (Guilkey and Weiss, 2003). The MPM is a mixed method combining the Eulerian and Lagrangian descriptions of the material. The Lagrangian description consists in representing each body by a collection of material points, and the Eulerian description is based on a background computational mesh. The information carried by material points is projected onto the background mesh, where equations of motion are solved. The mesh solution is then used to update the material points. The MPM brings together the advantages of Eulerian and Lagrangian methods by avoiding the distortion of Lagrangian mesh and tracking the boundaries of bodies. This method has also been applied to granular materials (Bardenhagen et al., 2000a; Cummins and Brackbill, 2002).

In particle systems, the kinematic constraints and stresses arising from unilateral contacts between particles and the bulk deformations of the particles are strongly coupled. While the contacts play the role of boundary conditions for the resolution of continuum equations in each particle, the evolution of contacts is determined by particle deformations. In MPM, the use of the same set of continuous shape functions in both mappings (the mapping from material points to mesh nodes and vice versa) naturally results in sticking (no interpenetration and no slip) contact scheme and thus no interpenetration occurs. With the use of a single-valued velocity to update the positions of material points, the sticking contact between two different bodies can be handled automatically at no additional computational cost using the original MPM, and no contact surface detection is required, but the contacting objects may not separate. For this reason, it is necessary to define different body velocities at the nodes and to implement frictional contact laws independently from MPM.

This issue has been addressed by several authors. Bardenhagen et al. (2000a, 2001) extended the original MPM to account for the unilateral nature of contact. In this algorithm, a contact occurs if the material points of different bodies are projected onto the same nodes of the background mesh, and the contact force is associated with the difference between the mass-center velocity of the particles and the node velocity, reflecting the constraints arising from contact. To release the noslip contact algorithm in MPM, (Hu and Chen, 2003) proposed a multi-mesh mapping scheme, i.e. material points of each body lie in an individual background mesh rather than in the common one. In this procedure, the normal velocity of a material points at the contact surface is calculated in the common background mesh while the tangential velocity is found from the corresponding information in respective individual meshes. In their scheme, normal acceleration is set to be the same if material points of different bodies are mapped on the same node. However, in this algorithm the friction between different bodies is ignored because the tangential velocities of different bodies are assumed to be independent. Another multi-mesh contact algorithm for MPM was proposed by Pan et al. (2008). In this approach, the contact condition is similar to that of Bardenhagen et al. (2000a, 2001). It allows for fast contact search between bolides. However, contrary to the previous contact algorithms, both normal and tangential velocities of each particle at the contact surface are calculated in the respective individual meshes.

In this paper, an implicit MPM procedure is proposed for the simulation of deformable particles in association with the $\mathrm{CD}$ method for the treatment of frictional contacts between particles. Our procedure is implemented in a manner that the contact variables (velocity, force, etc.) are computed simultaneously with bulk variables (stresses, strains, etc.). We validate our algorithm by considering simple single-particle problems involving one contact, namely Hertz contact between a particle and a rigid platen and rolling of a particle on an inclined plane. We briefly present these examples, and compare the 
simulation data with analytical predictions. We also present the uniaxial compaction of a small number of particles, and briefly investigate the compaction law and effect of friction. As we shall see, particle shape change may give rise to counterintuitive behaviors in granular materials.

\section{MPM: a finite element method with moving integration points}

In this section, we describe the basic formulation of the material point method. Indeed, MPM can be considered as a finite element method (FEM) with moving integration points (material points). In MPM, a body is discretized by a set of Lagrangian material points carrying all state variables such as stress and velocity field. The MPM algorithm uses an Eulerian mesh for solving the momentum equations. The material points are assigned fixed masses during computation so that the conservation of mass is satisfied implicitly. The momentum changes are interpolated from the grid to the material points, so that the total momentum is conserved (Guilkey and Weiss, 2003).

\subsection{Governing equations}

We consider a domain $\Omega$ in $\mathbb{R}^{D}, D$ being the domain dimension, with an external boundary $\partial \Omega$, describing a continuum body. Its conservation of mass is described by this continuity equation:

$$
\frac{\partial \rho(\mathbf{x}, t)}{\partial t}+\nabla(\rho(\mathbf{x}, t) \cdot \mathbf{v}(\mathbf{x}, t))=0 \quad \text { in } \Omega
$$

where $\rho(\mathbf{x}, t)$ indicates the material density and $\mathbf{v}(\mathbf{x}, t)$ denotes the velocity field at position $\mathbf{x}$ and time $t$.

In the context of the infinitesimal strain theory, conservation of linear momentum is defined as follows:

$$
\nabla \cdot \boldsymbol{\sigma}(\mathbf{x}, t)+\mathbf{b}(\mathbf{x}, t)=\rho(\mathbf{x}, t) \mathbf{a}(\mathbf{x}, t) \quad \text { in } \Omega,
$$

where $\boldsymbol{\sigma}(\mathbf{x}, t)$ is the Cauchy stress tensor, $\mathbf{b}(\mathbf{x}, t)$ represents the body force and $\mathbf{a}(\mathbf{x}, t)$ denotes the acceleration field at position $\mathbf{x}$ and time $t$.

This continuum body is subjected to prescribed displacements and forces on the disjoint complementary parts of the boundary $\partial \Omega_{u}$ (the Dirichlet boundaries) and $\partial \Omega_{f}$ (the Neumann boundaries), respectively. The boundary conditions are then defined by

$$
\begin{cases}\mathbf{u}(\mathbf{x}, t)=\hat{\mathbf{u}}(t) & \text { on } \partial \Omega_{u}, \\ \boldsymbol{\sigma}(\mathbf{x}, t) \cdot \mathbf{n}=\mathbf{f}(t) & \text { on } \partial \Omega_{f}\end{cases}
$$

where $\mathbf{u}(\mathbf{x}, t)$ and $\hat{\mathbf{u}}(t)$ are the displacement field and the prescribed displacement, respectively. In Eq. (3), $\mathbf{n}$ is the outward unit normal vector to $\partial \Omega$ and $\mathbf{f}(t)$ is a prescribed load.

The continuity equation (1) and the momentum equation (2) must be supplemented with a constitutive relationship which is assumed here to be linear, homogeneous, isotropic and elastic:

$$
\boldsymbol{\sigma}(\mathbf{x}, t)=\mathbb{C}: \boldsymbol{\epsilon}(\mathbf{x}, t),
$$

where $\mathbb{C}$ refers to fourth-order elastic tensor and $\boldsymbol{\epsilon}$ is the strain tensor $\left(\boldsymbol{\epsilon}=\frac{1}{2}\left(\nabla \mathbf{u}+\nabla \mathbf{u}^{T}\right)\right.$; superscript $T$ denotes the transpose of a matrix). It is worth noting that any other constitutive law (including inelastic behaviors such as plastic deformations) can be used in this context; but here, to simplify the presentation, the above constitutive relationship is considered.

\subsection{Variational and spatial discretized forms of the governing equations}

In the framework of the finite element method, the body is divided into infinitesimal mass elements to which a constant mass amount is allocated for all times; however, in MPM, these elements are represented by material points with fixed mass. Hence, since the material point mass is considered to be fixed in the MPM, mass conservation relation (1) is automatically satisfied. Then, the weak form associated with the momentum equation (2) and the boundary conditions (3) can be written as follows:

Find $\mathbf{u} \in \mathcal{S}(\Omega), \forall \delta \mathbf{u} \in \mathcal{S}_{0}(\Omega)$ and $\mathbf{u}=\hat{\mathbf{u}}$, on $\partial \Omega_{u}$ such that

$$
\int_{\Omega} \boldsymbol{\sigma}: \delta \boldsymbol{\epsilon} \Omega+\int_{\Omega} \rho \mathbf{a} \cdot \delta \mathbf{u} \mathrm{d} \Omega=\int_{\Omega} \mathbf{b} \cdot \delta \mathbf{u} \mathrm{d} \Omega+\int_{\partial \Omega f} \mathbf{f} \cdot \delta \mathbf{u} \mathrm{d} \Gamma
$$

where $\mathcal{S}(\Omega)$ denotes the space of sufficiently regular displacements and $\mathcal{S}_{0}(\Omega)=\left\{\delta \mathbf{u} \in \mathcal{S}(\Omega), \delta \mathbf{u}=0\right.$ on $\left.\partial \Omega_{u}\right\}$ is the corresponding space of suitable variations with vanishing values on the Dirichlet boundary.

The MPM can be considered as a modification of the classical finite element method. The classic nodal force and mass matrix integrals for the FEM grid are defined in a usual FEM manner. Hence, the weak form relation (5) can be rewritten as follows: 


$$
\sum_{e=1}^{N_{e}} \int_{\Omega^{e}} \boldsymbol{\sigma}: \delta \boldsymbol{\epsilon} \mathrm{d} \Omega+\sum_{e=1}^{N_{e}} \int_{\Omega^{e}} \rho \mathbf{a} \cdot \delta \mathbf{u} \mathrm{d} \Omega=\sum_{e=1}^{N_{e}} \int_{\Omega^{e}} \mathbf{b} \cdot \delta \mathbf{u} \mathrm{d} \Omega+\int_{\partial \Omega f} \mathbf{f} \cdot \delta \mathbf{u} \mathrm{d} \Gamma,
$$

where $\Omega^{e}$ represents the domain occupied by the eth element and $N_{e}$ is the number of elements. In the context of the MPM, the material points serve as the integration points to compute the FEM integrals. However, in standard FEM, the locations of the integration points and their weights are chosen to optimize the integration accuracy for a given set of interpolation functions. The MPM then discretizes these integrals through the use of a Dirac delta function by considering a fixed material point mass. In this context, for example, the density can be then discretized for an element as follows:

$$
\rho(\mathbf{x}, t)=\sum_{p=1}^{N_{p}^{e}} \rho_{p} \delta\left(\mathbf{x}-\mathbf{X}_{p}(t)\right),
$$

where $\rho_{p}$ and $\mathbf{X}_{p}(t)$ refer to material point density and position respectively. $\delta$ is the Dirac delta function and $N_{p}^{\mathrm{e}}$ is the number of the material points for an element. Note that the material point density is equal to $\rho_{p}=m_{p} / V_{p}$ with material point mass $m_{p}$ and volume $V_{p}$.

The displacement $\mathbf{u}_{p}$ and the strain $\boldsymbol{\epsilon}_{p}$ at material point $\mathbf{X}_{\mathbf{p}}$ are expressed in function of the displacements at one element's nodes $\mathbf{u}_{\text {node }}^{e}$ called elementary nodal displacements. $\mathbf{u}_{p}$ and $\mathbf{u}_{\text {node }}^{e}$ are related through $\mathbf{N}_{p}$ called interpolation matrix or shape function matrix at $\mathbf{X}_{\mathbf{p}}\left(\mathbf{u}_{p}=\mathbf{N}_{p} \mathbf{u}_{\text {node }}^{e}\right.$ ). In this context, $\mathbf{G}_{p}$ which links the stain $\boldsymbol{\epsilon}_{p}$ to the nodal displacements $\mathbf{u}_{\text {node }}^{e}$ can be easily defined ( $\boldsymbol{\epsilon}_{p}=\mathbf{G}_{p} \mathbf{u}_{\text {node }}^{e}$ ). Indeed, $\mathbf{G}_{p}$ is the gradient of the shape function $\mathbf{N}_{p}$. The virtual displacement $\delta \mathbf{u}_{p}$ and strain $\delta \boldsymbol{\epsilon}_{p}$ can be also defined in the same way $\left(\delta \mathbf{u}_{p}=\mathbf{N}_{p} \delta \mathbf{u}_{\text {node }}^{e}\right.$ and $\delta \boldsymbol{\epsilon}_{p}=\mathbf{G}_{p} \delta \mathbf{u}_{\text {node }}^{e}$ ). Therefore, the problem (6) in the discretized form can be written as follows by considering the contact interactions between several bodies:

$$
\mathbf{M a}_{\text {node }}(t)=\mathbf{F}^{\text {int }}(t)+\mathbf{F}^{\text {ext }}(t)+\mathbf{F}^{\mathrm{C}}(t)
$$

where $\mathbf{a}_{\text {node }}$ is the nodal acceleration, $\mathbf{F}^{\mathbf{C}}$ denotes the contact force which will be illustrated in Section 4 and

$$
\begin{aligned}
& \mathbf{M}=\sum_{e=1}^{N_{e}} \sum_{p=1}^{N_{p}^{e}} m_{p} \mathbf{N}_{p} \quad \text { lumped mass matrix, } \\
& \mathbf{F}^{\text {int }}=-\sum_{e=1}^{N_{e}} \sum_{p=1}^{N_{p}^{e}} \mathbf{G}_{p} \boldsymbol{\sigma}_{p} V_{p} \quad \text { internal force vector, } \\
& \mathbf{F}^{\text {ext }}=\sum_{e=1}^{N_{e}} \sum_{p=1}^{N_{p}^{e}} \mathbf{N}_{p} \mathbf{b}_{p}+\mathbf{F}^{S} \quad \text { sum of body forces and surface tractions, } \mathbf{F}^{\mathrm{S}} .
\end{aligned}
$$

Finally, since there are generally more material points than grid nodes, a weighted squares approach is used to determine nodal velocities $\mathbf{v}_{\text {node }}$ from the material point velocities $\mathbf{v}_{p}$. So, the nodal velocities are obtained by solving the relation:

$$
\mathbf{P}_{\text {node }}=\mathbf{M} \mathbf{v}_{\text {node }}=\sum_{e=1}^{N_{e}} \sum_{p=1}^{N_{p}^{e}} m_{p} \mathbf{N}_{p} \mathbf{v}_{p},
$$

where $\mathbf{P}_{\text {node }}$ is the nodal momentum.

\section{MPM: an implicit-type formalism}

We propose a MPM approach with an implicit time integration based on Guilkey and Weiss (2003) in this section. In the context of the implicit resolution, to advance the solution of (8) from $t$ to $t+\Delta t$, we consider that $\mathbf{F}^{\text {ext }}(t+\Delta t)$ is known. Moreover, the trapezoidal rule is used to advance the grid kinematics:

$$
\begin{aligned}
& \mathbf{u}_{\text {node }}(t+\Delta t)=\frac{\Delta t}{2}\left(\mathbf{v}_{\text {node }}(t)+\mathbf{v}_{\text {node }}(t+\Delta t)\right), \\
& \mathbf{v}_{\text {node }}(t+\Delta t)=\mathbf{v}_{\text {node }}(t)+\frac{\Delta t}{2}\left(\mathbf{a}_{\text {node }}(t)+\mathbf{a}_{\text {node }}(t+\Delta t)\right) .
\end{aligned}
$$

Note that since $\mathbf{u}_{\text {node }}(t+\Delta t)$ is in fact the grid displacement from $t$ to $t+\Delta t$, $\mathbf{u}_{\text {node }}(t)=0$. From Eqs. (10) and (11), the nodal acceleration at time $t+\Delta t$ can be obtained as

$$
\mathbf{a}_{\text {node }}(t+\Delta t)=\frac{4}{\Delta t^{2}} \mathbf{u}_{\text {node }}(t+\Delta t)-\frac{4}{\Delta t} \mathbf{v}_{\text {node }}(t)-\mathbf{a}_{\text {node }}(t) .
$$

To evaluate the material point volume $V_{p}$ change from $t$ to $t+\Delta t$, by considering the mass conservation equation (1), the 
trapezoidal rule and the fact that the material point mass $\left(m_{p}\right)$ is fixed, we get

$$
V_{p}(t+\Delta t)=V_{p}(t) \frac{1+\frac{\Delta t}{2} \nabla \cdot \mathbf{v}_{p}(t)}{1+\frac{\Delta t}{2} \nabla \cdot \mathbf{v}_{p}(t)-\operatorname{Tr}\left(\Delta \epsilon_{p}(t+\Delta t)\right)},
$$

where $\operatorname{Tr}(\cdot)$ is the trace of a matrix and

$$
\Delta \boldsymbol{\epsilon}_{p}(t+\Delta t)=\frac{1}{2}\left[\nabla \mathbf{u}_{p}(t+\Delta t)+{ }^{t} \nabla \mathbf{u}_{p}(t+\Delta t)\right]=\mathbf{G}_{p} \mathbf{u}_{\text {node }}^{e}(t+\Delta t) .
$$

In the framework of an incremental-iterative resolution algorithm, a new estimation of $\mathbf{u}_{\text {node }}$ at iteration $k, \mathbf{u}_{\text {node }}^{k}(t+\Delta t)$, is obtained by adding $\Delta \mathbf{u}_{\text {node }}^{k}$, the incremental displacement, to the previous estimated displacement:

$$
\mathbf{u}_{\text {node }}^{k}(t+\Delta t)=\mathbf{u}_{\text {node }}^{k-1}(t+\Delta t)+\Delta \mathbf{u}_{\text {node }}^{k}
$$

To obtain $\Delta \mathbf{u}_{\text {node }}^{k}$, at iteration $k$ we solve:

$$
\mathbf{K}^{k-1} \Delta \mathbf{u}_{\text {node }}^{k}=\mathbf{R}^{k},
$$

where $\mathbf{K}$ is the stiffness matrix and $\mathbf{R}$ refers to the residual term. This equation is the incremental form of relation (8). The terms $\mathbf{K}$ and $\mathbf{R}$ are defined in Appendix A.

The objective of this incremental algorithm is to find a nodal displacement $\mathbf{u}_{\text {node }}(t+\Delta t)$ that minimizes the residual term, R. So, as in Guilkey and Weiss (2003), we introduce two convergence criteria:

$$
C_{1}=\frac{\left\|\Delta \mathbf{u}_{\text {node }}^{k}\right\|}{\left\|\Delta \mathbf{u}_{\text {node }}^{\max }\right\|}<\epsilon_{1} \quad \text { and } \quad C_{2}=\frac{\left\|\Delta \mathbf{u}_{\text {node }}^{k} \mathbf{R}^{k}\right\|}{\left\|\Delta \mathbf{u}_{\text {node }}^{1} \mathbf{R}^{1}\right\|}<\epsilon_{2},
$$

where $\epsilon_{1}$ and $\epsilon_{2}$ are tolerance parameters on velocities and energy, respectively, $\|\cdot\|$ is the norm operator, $\left\|\Delta \mathbf{u}_{\text {node }}^{\max }\right\|$ denotes the maximum value of the norm of the incremental displacement and $\left\|\Delta \mathbf{u}_{\text {node }}^{1} \mathbf{R}^{1}\right\|$ indicates initial value of the inner product of the incremental displacement and the residual term.

In principal, a single criterion is sufficient for the convergence of all fields. Nevertheless, the use of two criteria allows for a better control of convergence as all fields do not converge with the same rate. One example is shown in Fig. 1, for the case of the uniaxial compaction of a packing of soft particles, discussed in detail in Section 5.3. The number of iterations for convergence is determined for a fixed value $\epsilon_{1}=10^{-1}$ as $\epsilon_{2}$ is varied from $10^{-3}$ down to $10^{-6}$ (dashed line) and for a fixed value $\epsilon_{2}=10^{-3}$ as $\epsilon_{1}$ is varied from $10^{-1}$ down to $10^{-3}$ (full line). In both cases, the number of iterations increases almost logarithmically as the convergence criterion declines. We see that the convergence criterion $C_{1}$ is more sensitive than $C_{2}$. However, the computation time per time step depends on the number of iterations, number of material points (or background mesh nodes for a given number of material points per mesh) and number of contacts. On a standard workstation (CPU speed of about $2 \mathrm{GHz}$ ), the computation time for uniaxial compaction presented in Section 5.3 with 9150 material points is about $2.5 \times 10^{-5} \mathrm{~s}$ per time step and per degree of freedom for $\epsilon_{1}=10^{-1}$, which requires about 7 iterations per time step and corresponds to $\epsilon_{2}=10^{-3}$.

\section{MPM: contact dynamics}

In dealing with contacts between deformable bodies, the contact forces $\mathbf{F}^{\mathrm{C}}$ need to be calculated by means of a contact algorithm accounting for the condition of non-interpenetration of matter, i.e. unilateral contact constraint, as well as the Coulomb friction law. In a discretized scheme, two bodies will interact at several contact points depending on their degree

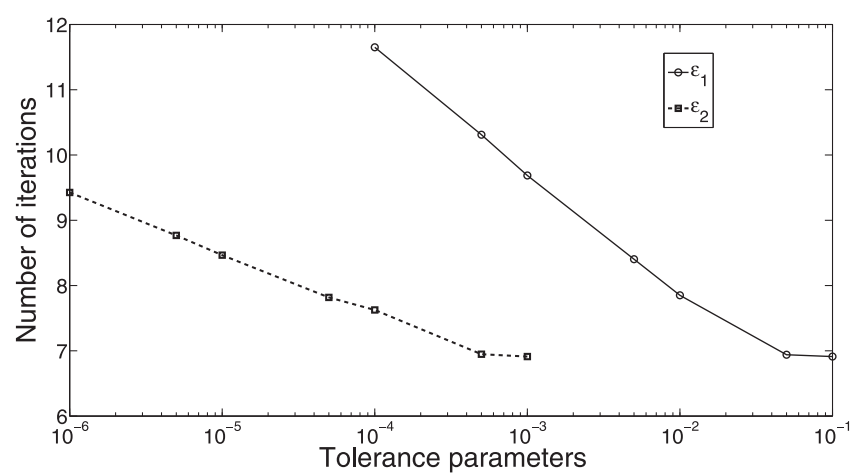

Fig. 1. Number of iterations as a function of tolerance parameters $\epsilon_{1}$ and $\epsilon_{2}$; see text. 


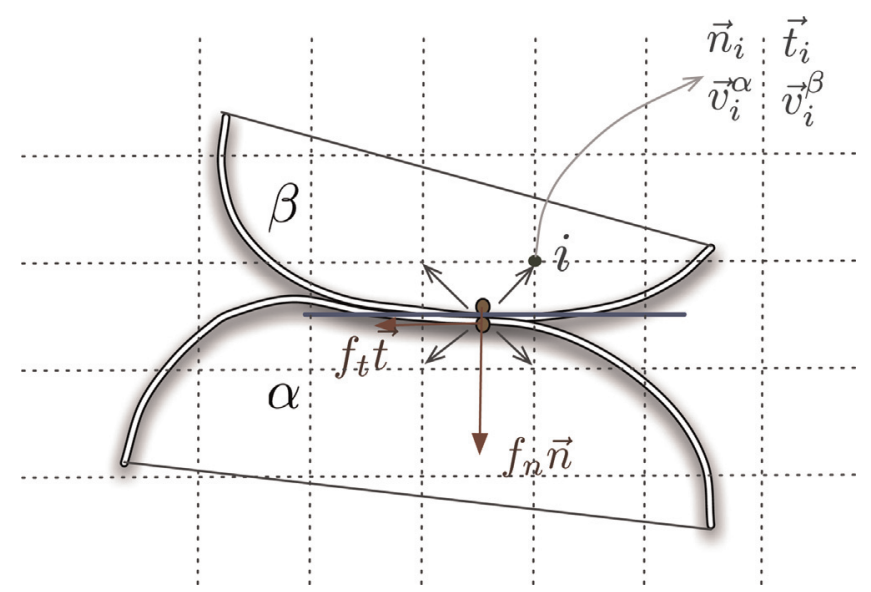

Fig. 2. Geometry of contact between two soft particles discretized in multi-mesh MPM algorithm; see text.

of deformation and spatial resolution used, and the resolution method in MPM does not allow for overlaps and slipping between bodies. Indeed, the use of the same set of continuous shape functions in both mappings (the mapping from material points to mesh nodes and vice versa) results naturally in a no-overlap no-slip contact scheme when the contacts are treated on common nodes between the two bodies (Bardenhagen et al. 2000a,b, 2001). However, a multi-mesh mapping can be used to implement not only the condition of no-overlap but also contact laws such as the friction Coulomb law or adhesion laws at the contact points (Hu and Chen, 2003). Several authors have extended the original MPM to account for frictional contacts between deformable solid bodies by introducing a multi-mesh environment (Hu and Chen, 2003; Pan et al., 2008). In these models, the contact variables are computed simultaneously with bulk stresses and strains. But the contacts are treated on the basis of a regularized scheme with an explicit time-stepping scheme.

The Contact Dynamics (CD) method is a general approach for the treatment of frictional contacts without regularization. This method was pioneered by a mathematical formulation of nonsmooth mechanics by Moreau (1988) and then extensively used for the simulation of granular materials with rigid grains (Radjai et al., 1996; Unger et al., 2002). Detailed descriptions of the foundations and algorithmic aspects of CD can be found in the literature (Moreau, 1994; Jean, 1995; Radjai and Richefeu, 2009). This method is based on an implicit time-stepping scheme and formulated in terms of grain velocities, which may undergo jumps as a result of collisions and the non-smooth feature of the Coulomb friction law. The use of the implicit MPM scheme in this paper was thus motivated by the implicit nature of the time-stepping procedure in the $\mathrm{CD}$ method. However, the $\mathrm{CD}$ method is generally associated with perfectly rigid grains, and thus the resolution method should be adapted to the grains discretized by material points.

Let us consider two deformable bodies denoted $\alpha$ and $\beta$; see Fig. 2. In the context of multi-mesh algorithm, each body maps in its proper background mesh. A contact point at the interface between the two bodies may be treated by introducing a common background mesh with the same type of grids for the transfer of nodal quantities from proper meshes to the common mesh. The contact points between the bodies $\alpha$ and $\beta$ are treated at the neighboring nodes belonging to the common background mesh. Their nodal values involve contributions from the two bodies. At a potential contact node $i$, a normal unit vector $\mathbf{n}_{i}$, oriented from body $\beta$ to body $\alpha$ is defined by the gradient of the nodal mass in the individual body (Bardenhagen et al., 2001; Huang et al., 2011):

$$
\mathbf{n}_{i}=\frac{\hat{\mathbf{n}}_{i}^{\beta}-\hat{\mathbf{n}}_{i}^{\alpha}}{\left|\hat{\mathbf{n}}_{i}^{\beta}-\hat{\mathbf{n}}_{i}^{\alpha}\right|},
$$

with

$$
\hat{\mathbf{n}}_{i}^{\alpha}=\frac{\sum_{p=1}^{N_{p}^{e}} \mathbf{G}_{p} m_{p}^{\alpha}}{\left|\sum_{p=1}^{N_{p}^{e}} \mathbf{G}_{p} m_{p}^{\alpha}\right|} \quad \text { and } \quad \hat{\mathbf{n}}_{i}^{\beta}=\frac{\sum_{p=1}^{N_{p}^{e}} \mathbf{G}_{p} m_{p}^{\beta}}{\left|\sum_{p=1}^{N_{p}^{e}} \mathbf{G}_{p} m_{p}^{\beta}\right|},
$$

where $m_{p}^{\alpha}$ and $m_{p}^{\beta}$ represent the mass of material point $p$ in bodies $\alpha$ and $\beta$, respectively. Moreover, a tangential unit vector $\mathbf{t}_{i}$ is defined by

$$
\mathbf{t}_{i}=\frac{\left(\mathbf{v}_{i}^{\alpha}-\mathbf{v}_{i}^{\beta}\right)-\left(\mathbf{v}_{i}^{\alpha}-\mathbf{v}_{i}^{\beta}\right) \cdot \mathbf{n}_{i} \mathbf{n}_{i}}{\left|\left(\mathbf{V}_{i}^{\alpha}-\mathbf{v}_{i}^{\beta}\right)-\left(\mathbf{v}_{i}^{\alpha}-\mathbf{v}_{i}^{\beta}\right) \cdot \mathbf{n}_{i} \mathbf{n}_{i}\right|}
$$

As long as the relative normal velocity $v_{n}$ remains positive, 

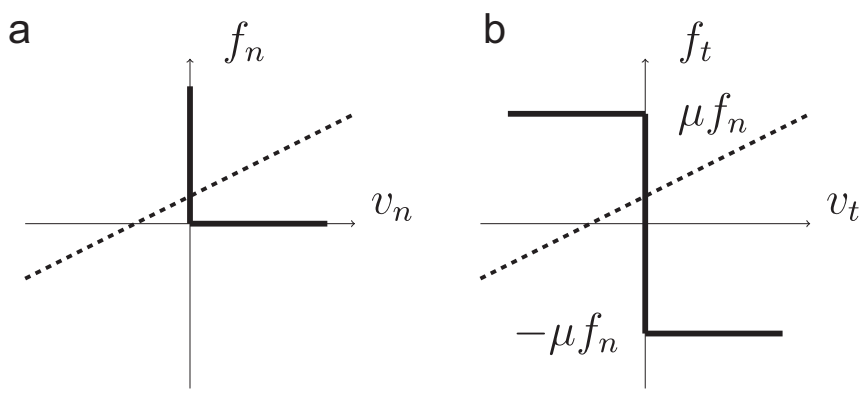

Fig. 3. Contact conditions: (a) Velocity-Signorini complementarity condition as a graph relating the normal relative velocity $v_{n}$ and normal force $f_{n}$; (b) Coulomb friction law as a graph relating the tangential velocity $v_{t}$ and friction force $f_{t} ; \mu$ is the coefficient of friction. The dashed lines represent linear relations representing the equations of dynamics.

$$
v_{n}=\left(\mathbf{v}_{i}^{\alpha}-\mathbf{v}_{i}^{\beta}\right) \cdot \mathbf{n}_{i}>0
$$

the normal force $f_{n}$ is identically zero. But when $v_{n}=0$, a non-negative (repulsive) normal force $f_{n}$ is mobilized at the contact node. These conditions define the velocity-Signorini complementarity condition as shown in Fig. 3(a) (Jean, 1995; Brogliato, 1999).

In order to solve the equations of motion together with the velocity-Signorini condition at all potential contact nodes, it is necessary to express the equations of motion in the contact variables $f_{n}$ and $v_{n}$. Such a relation can be obtained by combining the equations of motion at the common node:

$$
\begin{aligned}
& m_{i}^{\alpha} \mathbf{a}_{i}^{\alpha}=\mathbf{f}_{i}^{\alpha, \text { int }}+\mathbf{f}_{i}^{\alpha, \text { ext }}+\mathbf{f}_{i}^{\mathrm{C}, \alpha \beta}+\sum_{\gamma} \mathbf{f}_{i}^{\mathrm{C}, \alpha \gamma}, \\
& m_{i}^{\beta} \mathbf{a}_{i}^{\beta}=\mathbf{f}_{i}^{\beta, \text { int }}+\mathbf{f}_{i}^{\beta, \text { ext }}-\mathbf{f}_{i}^{\mathrm{C}, \alpha \beta}-\sum_{\gamma} \mathbf{f}_{i}^{\mathrm{C}, \gamma \beta},
\end{aligned}
$$

where $\sum_{\gamma} \mathbf{f}_{i}^{\mathrm{C}, \alpha \gamma}$ and $\sum_{\gamma} \mathbf{f}_{i}^{\mathrm{C}, \gamma \beta}$ are contact forces of other bodies at this common node. From the above equations, it is easy to get a linear relation between $f_{n}\left(f_{n}=\mathbf{f}_{i}^{\mathrm{C}, \alpha \beta} \cdot \mathbf{n}_{i}\right)$ and $v_{n}$ :

$$
f_{n}=\frac{2}{\Delta t} \frac{m_{i}^{\alpha} m_{i}^{\beta}}{m_{i}^{\alpha}+m_{i}^{\beta}} v_{n}+k_{n},
$$

where $k_{n}$ is an offset force which depends on other contact forces exerted by the neighboring bodies of $\alpha$ and $\beta$.

As shown in Fig. 3(a), Eq. (23) intersects the Signorini graph at a single point due to the positivity of the mass. Since the point of intersection depends on the offset $k_{n}$ and thus other contact nodal forces, the determination of nodal forces and velocities requires an iteration process between the values of $k_{n}$ and $f_{n}$ or $v_{n}$.

In a similar vein, the Coulomb law of dry friction is a complementarity relation between the friction force $f_{t}$ and the relative tangential velocity $v_{t}\left(v_{t}=\left(\mathbf{v}_{i}^{\alpha}-\mathbf{v}_{i}^{\beta}\right) \cdot \mathbf{t}_{i}\right)$ at the contact node; Fig. 3(b). Like the Signorini graph, the Coulomb law cannot be reduced to a single-valued function. The friction force and the tangential velocity can be determined by expressing the equations of motion in terms of contact variables $f_{t}$ and $v_{t}$ :

$$
f_{t}=\frac{2}{\Delta t} \frac{m_{i}^{\alpha} m_{i}^{\beta}}{m_{i}^{\alpha}+m_{i}^{\beta}} v_{t}+k_{t} .
$$

This linear relation can be intersected with the Coulomb graph to compute the friction force $f_{t}$ simultaneously at all contact nodes within the same iterative process used to compute the normal force through successive corrections.

It is important to note that the contact nodes are fixed at the beginning of each time step. Hence, the iterative process involves only the forces and relative velocities, which are the main unknowns of the contact problem. The positions are updated as a function of calculated velocities at the end of the time step. The convergence to the solution both for contact forces and internal stresses is smooth, and a high precision may be achieved depending on the convergence criterion. The implicit-type time stepping endows the method with good convergence properties. Besides the material behavior of the particles, which may involve viscous damping or plastic dissipation of energy, a major source of dissipation in granular dynamics is inelastic collisions and sliding friction between particles. The introduction of restitution coefficients in the CD formalism is presented in Radjai and Richefeu (2009). Note that, when contact dynamics equations are solved together with MPM equations in the bulk of the particles, the convergence criteria of equation (17) are used. The convergence is checked for contact interactions within the same iteration loop as bulk fields. 


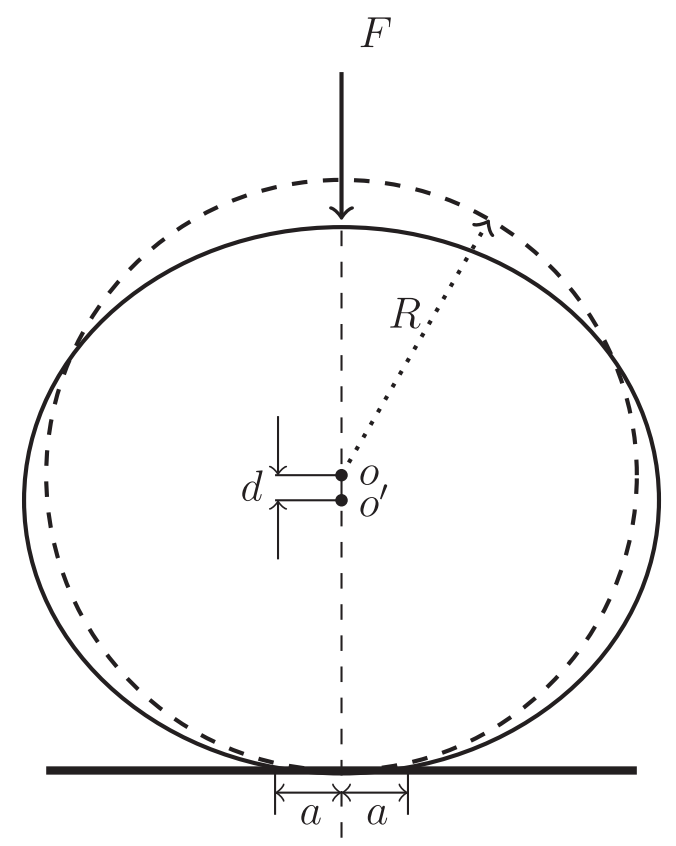

Fig. 4. Contact geometry between a disk and a rigid plate.

\section{Numerical examples}

In this section, we study the accuracy and efficiency of the proposed algorithm through several contact problems. The results are presented through three main examples. The first one concerns the Hertzian contact. It is used to show the validity of our contact algorithm in MPM. The second example deals with the rolling of an elastic disk on an inclined plane where we would like to reproduce the analytical rolling behavior at high rigidity. In the third example, we study the compaction of a packing of elastic grains in order to show the effectiveness of the proposed algorithm to deal with softparticle assemblies.

These examples have been treated by means of two-dimensional MPM in plane stress conditions. The computation domain has been meshed with four-node quadrangular elements, and an initial distribution of four material points per element was used. In all the tests presented below, we set $\epsilon_{1}=10^{-1}$ and $\epsilon_{2}=10^{-3}$; see Eq. (17).

\subsection{Hertzian contact}

We consider here the contact between an elastic disk and a rigid wall. The elastic disk may be modeled as a cylinder of unit length and radius $R$ as shown in Fig. 4. Its contact with the wall occurs over a unit strip of width $2 a$. In the absence of friction force between the disk and the wall, it can be shown that the contact force $F$ is proportional to the displacement $d$ of the center of the cylinder (see Johnson, 1999):

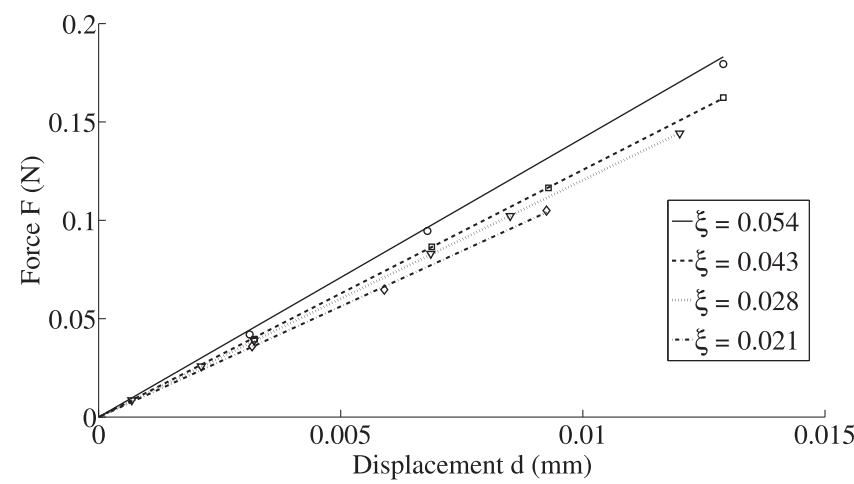

Fig. 5. Force-displacement plots of $2 \mathrm{D}$ Hertz contact for different levels of relative spatial resolution $\xi$. 


$$
F=\frac{\pi}{4} E^{*} d
$$

where $E^{*}$ is the effective elastic modulus defined as $E^{*}=E /\left(1-\nu^{2}\right)$ with $E$ being Young's modulus and $\nu$ Poisson's ratio. Note that, in contrast to the 3D case (contact between two spheres), in 2D the particle radius $R$ does not enter the expression of $F$.

We performed MPM simulations of this $2 \mathrm{D}$ contact problem with $R=0.5 \mathrm{~mm}$ compressed between two rigid walls. The bottom wall is fixed and the top wall moves downwards at a constant velocity of $0.05 \mathrm{~m} / \mathrm{s}$. The time step is $\Delta t=0.1 \mu \mathrm{s}$. The gravitational acceleration is set to be zero in order to avoid stress gradients. Young's modulus, Poisson's ratio and density of the disk are set to $E=10 \mathrm{MPa}, \nu=0.45$ and $\rho=990 \mathrm{~kg} / \mathrm{m}^{3}$, respectively. The simulation was repeated for different levels of spatial resolution. The continuity of stress and strain fields inside the particles depends on the number of material points used to discretize the particle. For this reason, we define the relative spatial resolution $\xi$ by

$$
\xi=\frac{\Delta r}{R},
$$

where $\Delta r$ is the mean distance between material points.

Fig. 5 shows the normal contact force $F$ as a function of displacement $d$ of the center of the cylinder for four values of $\xi$. We observe a linear relation between force $F$ and displacement $d$ as predicted by the analytical expression (25), but the effective elastic modulus $E^{*}$, given by the slope, depends on $\xi$. In finite element simulations, the finite spatial resolution is known to affect the effective elastic properties, and an insufficient number of elements or meshes in a solid region or at an interface may not represent correctly continuum elasticity. But the elastic moduli are generally affine functions of the spatial resolution (Roberts and Teubner, 1995; Gatta et al., 2005; Affes et al., 2012). Hence, this property allows one to obtain an "exact" values of elastic moduli by extrapolation from data points for several levels of spatial resolution to the limit of infinitely high resolution. Fig. 6 shows the effective elastic modulus $E^{*}$ as a function of $\xi$ for our 2D contact problem. We see that the evolution of $E^{*}$ is indeed linear as a function of $\xi$ and it tends to the analytical value $E_{\text {analytic }}^{*} \simeq 12.5 \mathrm{MPa}$ obtained from Eq. (25).

This example shows that our MPM contact algorithm can correctly reproduce the Hertz contact behavior in 2D with an increasingly accurate prediction of contact stiffness for increasing spatial resolution. It is important to note here that the elastic moduli in the bulk of the material correctly reflect the imposed elastic moduli even for a coarse discretization. The issue at hand in the contact problem is the same as in all heterogeneous or composite materials in which the "effective" properties strongly depend on the interfaces between different phases. The conditions required for the continuity of stress and strain fields at such interfaces are far strongest than those within each phase. In the Hertz contact, the stress field in the contact area is controlled by the curvature of the particle around the positions $a$ and $-a$ from the center of the contact zone (see Fig. 4), which clearly requires a very high resolution. For this reason, as long as small deformations are concerned, it is generally more convenient to use numerical methods based on the analytical solutions as is the case, for example, in DEM. This is no more the case at large deformations, for which the MPM/CD algorithm provides the proper simulation tool provided the effects of discretization are correctly handled.

\subsection{Inclined plane: rolling disk}

An elastic disk on an inclined rigid plane as shown in Fig. 7 is considered. This plane is inclined at an angle $\theta$ above the horizontal, with the gravity $g$ oriented vertically downwards. A rigid disk placed at zero velocity on an inclined rigid plane will roll and slip when $\tan \theta>3 \mu$ where $\mu$ is the dry coefficient of friction. Conversely, for $\tan \theta \leq 3 \mu$, the disk will roll without slipping. The $x$-component $x_{c m}(t)$ of the center-of-mass position of the rigid disk evolves as

$$
x_{c m}(t)= \begin{cases}x_{0}+\frac{1}{2} g t^{2}(\sin \theta-\mu \cos \theta) & \tan \theta>3 \mu \text { (roll and slip), } \\ x_{0}+\frac{1}{3} g t^{2} \sin \theta & \tan \theta \leq 3 \mu \text { (roll), }\end{cases}
$$

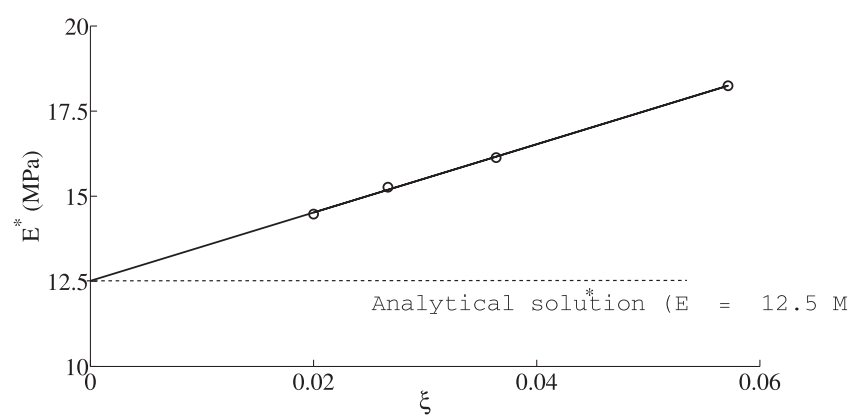

Fig. 6. Effective elastic modulus $\left(E^{*}\right)$ as a function of spatial resolution $\xi$. The analytical solution for given values of the parameters is displayed. 


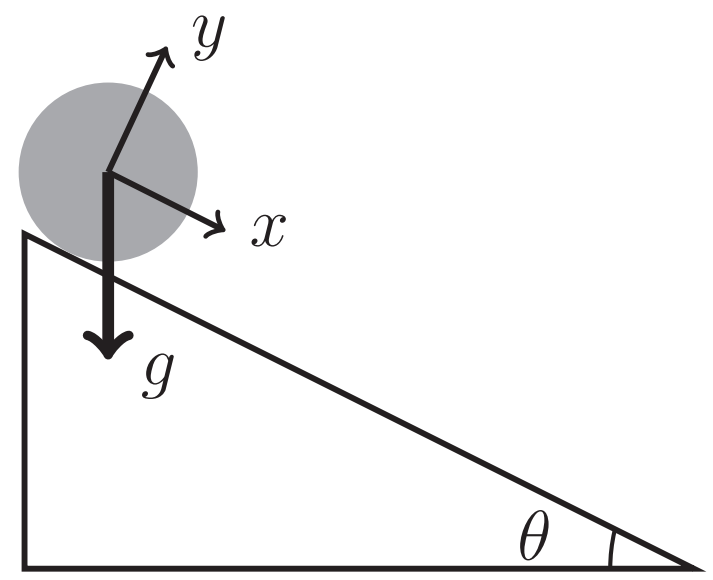

Fig. 7. Geometry for the simulation of a disk on an inclined plane.

where $x_{0}$ is the initial position.

In this test, we would like to show that MPM with frictional contact is able to reproduce the rolling behavior of the particle in the theoretical limit of very rigid particles. The kinematics of material points in a rolling particle is a harsh problem for MPM. Moreover, in the rigid limit particle deformations are small and a high resolution is needed to reproduce the strain and stress fields in the contact area. For the simulations, we used an elastic disk of radius $R=0.5 \mathrm{~mm}$ and an inclined rigid wall at an angle of $\pi / 4$. The gravitational acceleration is set to $10 \mathrm{~m} / \mathrm{s}^{2}$ and the time step is equal to $\Delta t=10 \mu \mathrm{s}$. The disk has Young's modulus $E=10 \mathrm{MPa}$, Poisson's ratio $\nu=0.45$ and density $\rho=990 \mathrm{~kg} / \mathrm{m}^{3}$. The same spatial resolution values as in the Hertz contact example were employed. Here, only the case of rolling without slipping is simulated by setting the coefficient of friction to $\mu=0.6$.

In Fig. 8, we compare the numerical results for the time evolution of the center-of-mass position of the disk obtained for different levels of spatial resolution with the analytical solution for an infinitely rigid disk. For all values of $\xi$, we observe a parabolic evolution of $x_{c m}$ with parameter $s=\left(x_{c m}-x_{0}\right) /\left(g t^{2} \sin \theta\right)$ converging towards the analytical value $1 / 3$ in agreement with Eq. (27), as shown in Fig. 9. The linear dependence of $s$ on spatial resolution is similar to that of the effective modulus in the Hertz contact problem, and it clearly reflects the local curvatures around the contact. The observed trend (decreasing acceleration for decreasing resolution) is consistent with the fact that the curvature is high at the limit points of the contact zone when spatial resolution is low. This leads to high stress concentration and numerical dissipation, which slows down the particle. Moreover, it is worth noting that as mentioned in the first example, as long as the simulations of the rigid bodies are concerned (i.e. when we have small deformations), the numerical methods based on the analytical solutions are more convenient. For example, in this example, if we freeze the internal degrees of freedom of the particle, we are left with the $C D$ method, which provides the exact solution without problem.

\subsection{Compaction of a packing of elastic grains}

Let us now consider a packing of 30 disks confined inside a rectangular box of width $L$ in which only the top wall is

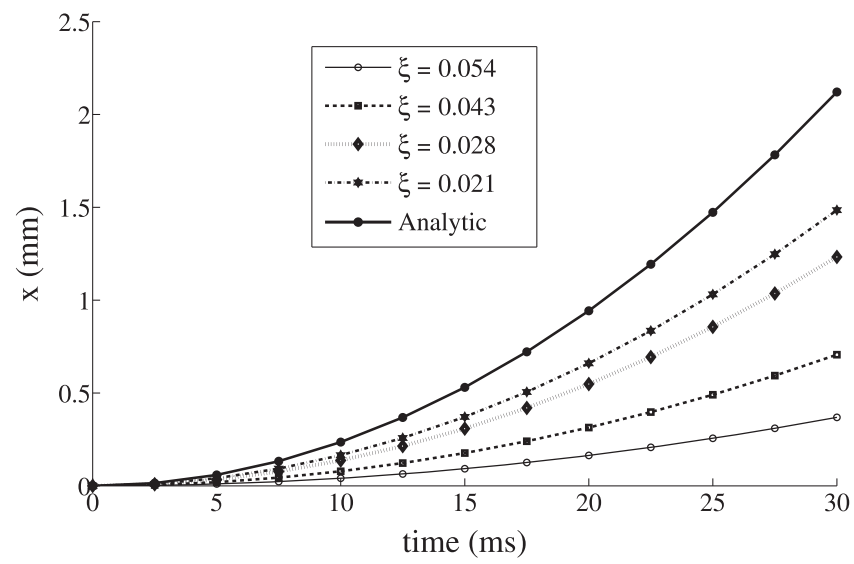

Fig. 8. The $x$-component of the center-of-mass of position of an elastic disk as a function of time for different levels of relative spatial resolution $\xi$. The bold line represents the analytical solution in the limit of an infinitely rigid disk. 


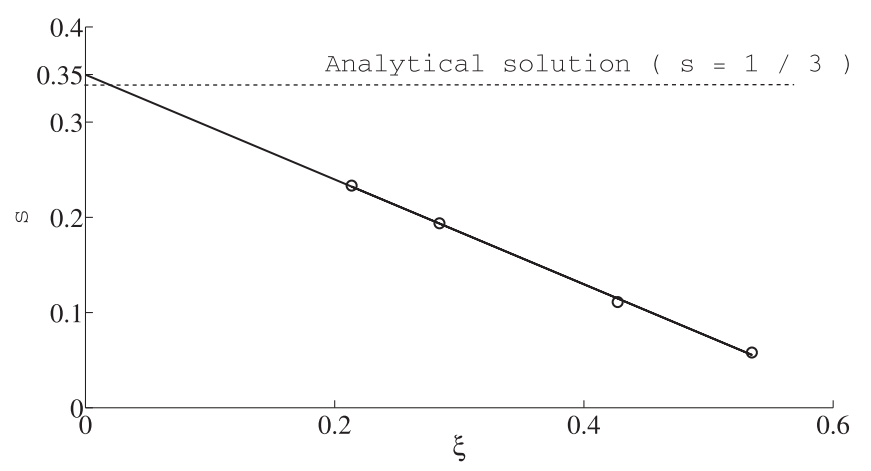

Fig. 9. Parameter $s=\left(x_{c m}-x_{0}\right) /\left(g t^{2} \sin \theta\right)$ as a function of relative spatial resolution $\xi$.

mobile. The initial configuration is prepared by means of DEM simulations. A small polydispersity is introduced in order to avoid long-range ordering. The box height and packing fraction at equilibrium are $h_{0}$ and $\phi_{0}$, respectively. The disks are initially compacted by exerting a stress $\sigma_{0}$ on the top wall. Starting with this configuration, we simulate the compaction process by moving the top wall downwards at constant velocity of $5 \mathrm{~m} / \mathrm{s}$ and a time step of $\Delta t=0.1 \mu \mathrm{s}$. Two simulations of compaction are analyzed below: (1) without friction and (2) with a coefficient of friction $\mu=0.5$ between the grains, and between the grains and the walls. The gravitational acceleration is set to be zero in order to avoid stress gradients. Young's modulus, Poisson's ratio and density of the particles are set to $E=10 \mathrm{MPa}, \nu=0.45$ and $\rho=990 \mathrm{~kg} / \mathrm{m}^{3}$, respectively.

The packing fraction is expected to increase due to three effects: (1) elastic volume change of the particles, (2) particle

a

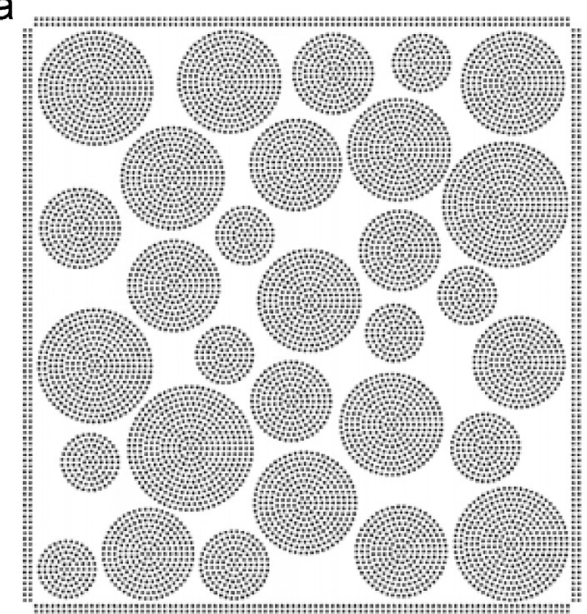

C

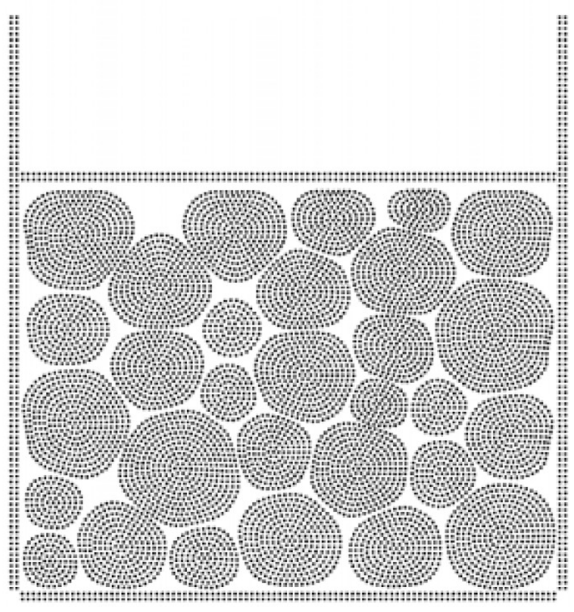

b

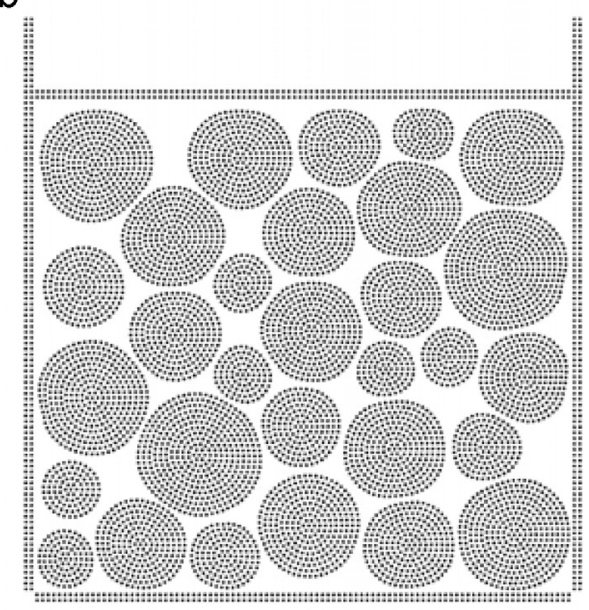

d

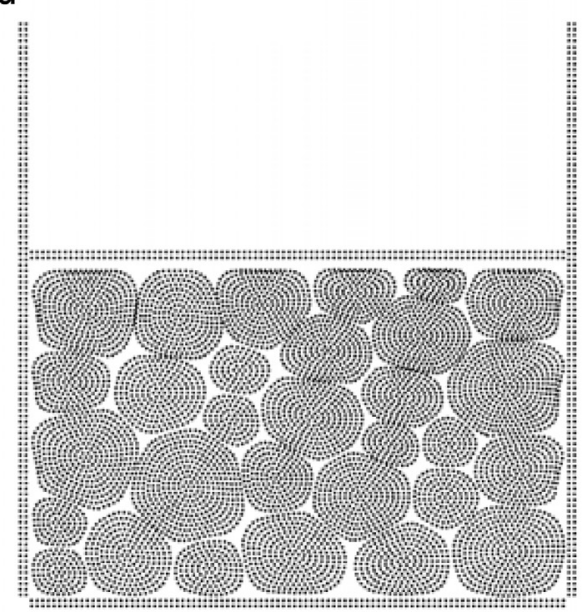

Fig. 10. Four snapshots of the compaction of a packing of elastic grains. Circle dots represent the material points. 


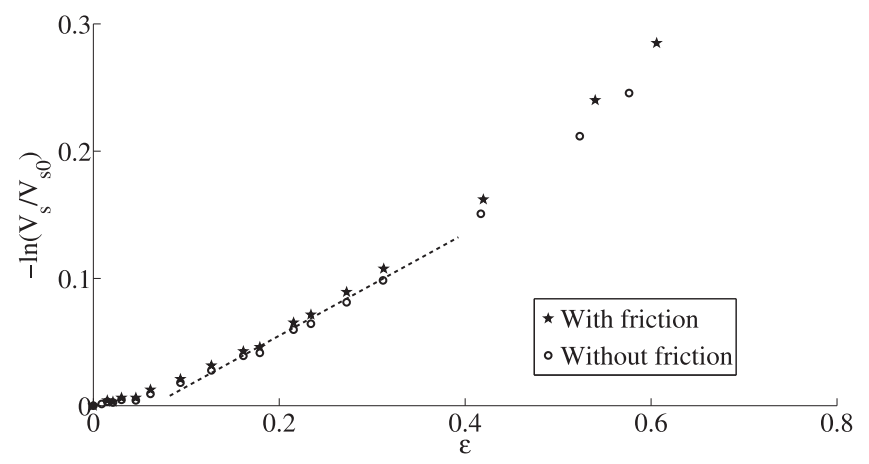

Fig. 11. Evolution of the total particles volume change as a function of the cumulative vertical strain $\varepsilon$. The dashed line represents the regime of contact deflections; see text.

rearrangements and (3) particle shape change. Only the latter may allow the packing fraction to considerably exceed the random close packing (RCP) limit. We are interested here in the mechanisms by which the particles exceed this limit and the evolution of the packing fraction with the compressive stress.

Fig. 10 displays four snapshots of the compaction test with $\mu=0$. The material points have been distributed so as to fit best to the initially circular shapes of the particles. The particle shape changes are clearly observed by following the positions of the material points. Note that the observed gaps between particles are a consequence of the meshing resolution, which may be increased to get a finer discretization of the contact zone. The snapshots show that, as the total volume decreases, new contacts are formed between particles, and the total contact area grows.

The total volume $V$ is the sum of the volume $V_{s}$ of particles and the volume $V_{v}$ of voids. Hence, the packing fraction $\phi=V_{s} /\left(V_{s}+V_{v}\right)$ varies due to both the volumetric particle deformation $\Delta V_{s} / V_{s}$ as a result of the elastic compressibility of particles and the variation of $V_{v}$ due to particle rearrangements and shape change. Since the width of the box is constant, we have

$$
\ln \left(\frac{V_{s}}{V_{s 0}}\right)=\ln \left(\frac{\phi}{\phi_{0}}\right)+\varepsilon
$$

where $\varepsilon \equiv \ln \left(1+\Delta h / h_{0}\right)$ is the true cumulative vertical strain. Fig. 11 shows the cumulative volume change of the particles $V_{S} / V_{s 0}$ as a function of $\varepsilon$ with and without friction. We see that the data for frictional and frictionless particles nearly coincide. The cumulative volume deformation $\ln \left(V_{s} / V_{s 0}\right)$ of the particles is small at the beginning of compression, indicating that the compaction is mainly governed by particle displacements. From $\varepsilon=0.1$ to $\varepsilon=0.3$ the volumetric deformation of the particles is nearly linear in $\varepsilon$ with a coefficient equal to Poisson's ratio $\nu=0.45$. This range corresponds to particle strains due to contact deflections, as in the Hertz regime. Beyond $\varepsilon=0.3$, the particle volume deformation grows at increasingly higher rate, which means that the deformation of the sample is increasingly governed by particle deformation while at the same time the void volume fraction decreases.

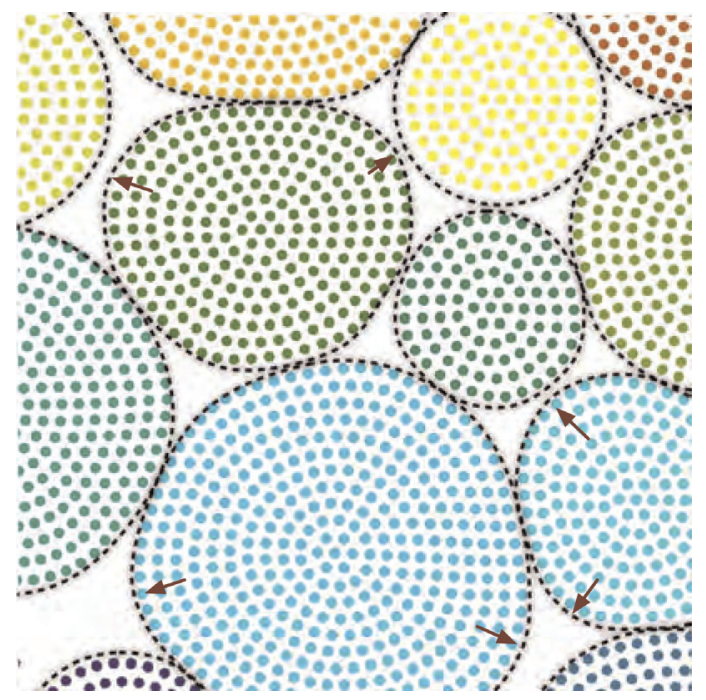

Fig. 12. A zoom on the deformed packing with particle borders and material points represented. The arrows indicate borders of large curvature. 


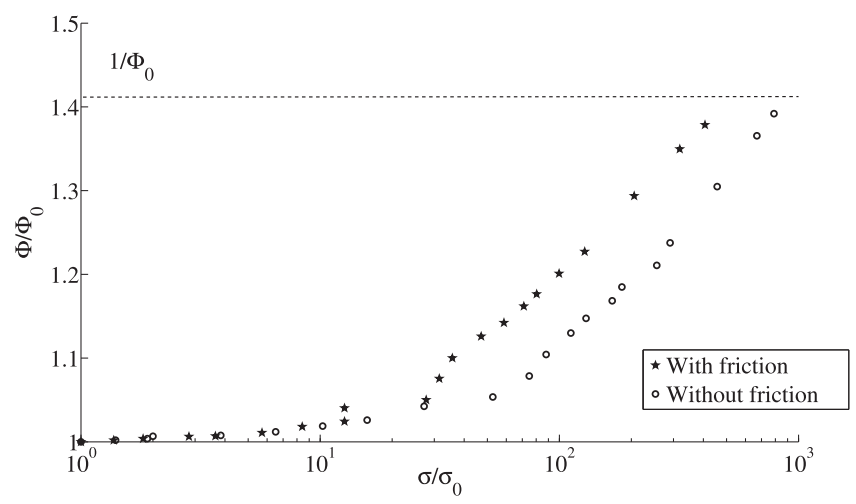

Fig. 13. Normalized packing fraction as a function of axial stress for frictionless and frictional particle packings.

Recent investigations show that the packing fraction increases with the deviation of particle shape from circular shape (Donev et al., 2007; Azéma and Radjai, 2010; Saint-Cyr et al., 2011; CEGEO et al., 2012). A generic parameter quantifying this deviation can be defined whatever the nature of deviation (degree of angularity, elongation etc.). Particles of non-circular shape can "overfill" the pores as a result of larger curvature at some parts of their boundary. Hence, as soft particles deform, a similar mechanism naturally leads to increasingly smaller voids and thus larger packing fraction. Fig. 12 displays an example of a deformed configuration where higher connectivity and packing fraction are reached as a result of particle shape change.

Fig. 13 shows the packing fraction $\phi$ as a function of the applied stress $\sigma$. Two regimes can clearly be distinguished. In the first regime, up to $\sigma \simeq 30 \sigma_{0}, \phi$ increases logarithmically with $\sigma$. In this range, the data nearly coincide for frictionless and frictional particles. The particle configuration evolves as a result of new contacts forming between particles and global rearrangements that are reflected in the irregularities and jumps observed on the points. In contrast, the connectivity of particles does not evolve in the second regime $\left(\sigma>30 \sigma_{0}\right)$. The evolution of $\phi$ is again linear as a function of the logarithm of $\sigma$ but at a higher rate. Both with friction and without friction, the ratio $\phi / \phi_{0}$ tends to $1 / \phi_{0} \simeq 1.42$, which is its highest possible value when the void volume $V_{v}$ vanishes.

It is also remarkable that, in this range, the packing fraction for frictional particles is above that for frictionless particles. This suggests that particle shape change is larger with friction. This is what we observe in Fig. 14 where two configurations are shown at the same level of deformation together with Von Mises stresses inside the particles. The friction between particles allows for stronger and more linear stress chains as compared to frictionless particles, where the particles need to be propped by lateral normal particles and forces. At the packing scale, this means that the ratio of the vertical stress to the horizontal stress in the case of frictional particles is higher than in the case of frictionless particles, where the stresses are nearly isotropic. Hence, in the frictional case, the higher stress ratio leads to more elongated particles and thus a larger packing fraction. However, given the small number of particles considered in these tests, we think that simulations with larger numbers of particles are necessary in order to analyze in more detail the effect of friction both between the grains and between the grains and lateral walls.

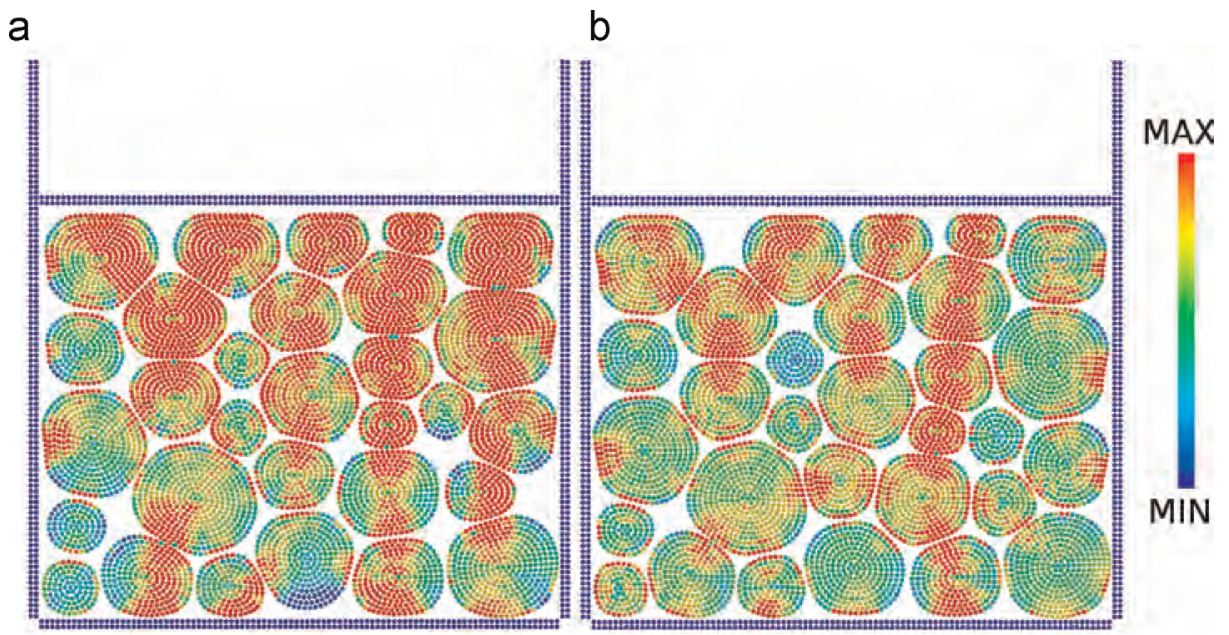

Fig. 14. Deformed packings at the same level of deformation with friction (a) and without friction (b). The Von Mises stress fields are represented in color code. (For interpretation of the references to color in this figure caption, the reader is referred to the web version of this paper.) 
It is worth noting that the logarithmic dependence of packing fraction with respect to the mean stress is commonly observed in soil mechanics. It is often attributed to particle fragmentation, which leads to progressive filling of the pore space between particles and hence the increase of $\phi$ with stress. In such a process, the stress field becomes increasingly homogeneous so that an increasingly larger stress is required to crush the particles. In the case of deformable particles, the "overfilling" of the pore space is due to shape change, with the effect of increasing the contact areas and hence the homogeneity of the stress field. In this way, as in the case of fragmentation, further increase of the packing fraction requires an increasingly larger stress.

\section{Conclusion}

In this paper, a new approach was proposed for the simulation of soft-particle systems. This approach combines an implicit formulation of the Material Point Method (MPM) for individual particles with the Contact Dynamics (CD) method for the treatment of frictional contacts. The realistic mechanical behavior of individual particles, including elastic and inelastic behaviors with the possibility of large deformations with or without rupture, can be taken into account due to MPM. Moreover, the $\mathrm{CD}$ allows us to deal with frictional contacts as unilateral interactions without regularization due to implicittype time integration. By construction, this algorithm is capable of handling arbitrary particle shapes with point-wise or extended contacts. Several 2D examples were presented to illustrate the potential of this approach to simulate contact interactions between soft particles and the effect of discretization. In particular, we showed that the numerical results tend to analytical solutions by increasing spatial resolution.

This approach provides an alternative to Discrete Element Methods (DEM) such as MD and CD with the advantage of allowing for realistic individual material behavior of the particles. The behavior of granular materials for rigid particles thus can be compared in detail with both $\mathrm{CD}$ (for the effect of particle deformation) and MD (for the effect of particle shape change). Such a validation is interesting, but it is obvious that, due to effect of finite spatial resolution, for small deformations it is generally more convenient to use numerical methods based on the analytical solutions as in DEM. Our MPM/ $\mathrm{CD}$ algorithm provides the proper simulation tool for larger deformations. It can be used to investigate the nonlinear effects arising from particle shape change and mitigate in more detail the extent to which analytical force laws can be used for the simulation of particle systems.

More fundamentally, this method can be used to investigate the mechanical behavior of very soft particles with the effect of shape change and space-filling well beyond the usual RCP packings. As an example, the uniaxial compaction of a small stack of elastic particles was simulated and the relationship between particle shape change and the evolution of the packing fraction was analyzed. It was shown that the packing fraction beyond the RCP state is a logarithmic function of the compressive stress. Interestingly, the friction appears to enhance the compressive stress, which leads to higher particle shape change and hence higher packing fraction.

This method opens broad prospects for realistic simulation of granular materials. It is obvious that the treatment of a large number of particles by this method requires large memory and fast processors, as well as further numerical developments such as a parallelization strategy. On the other hand, the extension of this approach to 3D is straightforward since both bulk and contact behaviors are handled at the nodes of a fixed background mesh.

\section{Acknowledgment}

Farhang Radjai would like to acknowledge the support of the ICoME2 Labex (ANR-11-LABX-0053) and the A*MIDEX projects (ANR-11-IDEX-0001-02) cofunded by the French programme Investissements dAvenir, managed by the ANR, the French National Research Agency.

\section{Appendix A. Definitions of $K$ and $R$}

The implicit integration in the context of MPM takes into account the discretized equation of the motion:

$$
\mathbf{M a}_{\text {node }}(t+\Delta t)=\mathbf{F}^{\text {int }}(t+\Delta t)+\mathbf{F}^{\text {ext }}(t+\Delta t) .
$$

By considering that the external force at time $t+\Delta t$ is known, $\mathbf{F}^{\text {ext }}(t+\Delta t)$, and by assuming an incremental-iterative Newton solution strategy, the linearized equation of motion at iteration $k$ is

$$
\mathbf{K}^{k-1} \Delta \mathbf{u}_{\text {node }}^{k}=\mathbf{R}^{k},
$$

where 


$$
\mathbf{K}^{k-1}=\left.\left(\mathbf{M} \frac{\partial \mathbf{a}_{\text {node }}\left(\mathbf{u}_{\text {node }}(t+\Delta t)\right)}{\partial \mathbf{u}_{\text {node }}(t+\Delta t)}-\frac{\partial \mathbf{F}^{\text {int }}\left(\mathbf{u}_{\text {node }}(t+\Delta t)\right)}{\partial \mathbf{u}_{\text {node }}(t+\Delta t)}\right)\right|_{\mathbf{u}_{\text {node }}(t+\Delta t)=\mathbf{u}_{\text {node }}^{k-1}(t+\Delta t)}
$$

$$
\mathbf{R}^{k}=\mathbf{F}^{\mathrm{ext}}(t+\Delta t)+\mathbf{F}^{\text {int }}{ }^{k-1}(t+\Delta t)-\mathbf{M a}_{\text {node }}^{k-1}(t+\Delta t)
$$

with

$$
\begin{aligned}
& \frac{\partial \mathbf{a}_{\text {node }}\left(\mathbf{u}_{\text {node }}(t+\Delta t)\right)}{\partial \mathbf{u}_{\text {node }}(t+\Delta t)}=\frac{4}{\Delta t^{2}}, \\
& \left.\frac{\partial \mathbf{F}^{\text {int }}\left(\mathbf{u}_{\text {node }}(t+\Delta t)\right)}{\partial \mathbf{u}_{\text {node }}(t+\Delta t)}\right|_{\mathbf{u}_{\text {node }}(t+\Delta t)=\mathbf{u}_{\text {node }}^{k-1}(t+\Delta t)}=\sum_{e=1}^{N e} \sum_{p=1}^{N e}\left\{V_{p}^{k-1}(t+\Delta t) \mathbf{G}_{p} \mathbf{C G}_{p}+\frac{V_{p}(t)}{1-\frac{\Delta t}{2} \nabla \cdot \mathbf{v}_{p}(t)} \mathbf{G}_{p} \boldsymbol{\sigma}_{p}^{k-1}(t+\Delta t) \mathbf{B} \mathbf{G}_{p}\right\}, \\
& \operatorname{Tr}\left(\Delta \boldsymbol{\epsilon}_{p}\right)=\mathbf{B G}_{p} \mathbf{u}_{n}^{e} .
\end{aligned}
$$

\section{References}

Affes, R., Delenne, J.Y., Monerie, Y., Radjaï, F., Topin, V., 2012. Tensile strength and fracture of cemented granular aggregates. Eur. Phys. J. E 35 , 117.

Agnolin, I., Roux, J.-N., 2007a. Internal states of model isotropic granular packings. I. Assembling process, geometry, and contact networks. Phys. Rev. E Stat. Nonlinear Soft Matter Phys. 76 (6-1), 061302.

Agnolin, I., Roux, J.-N., 2007b. Internal states of model isotropic granular packings. III. Elastic properties. Phys. Rev. E Stat. Nonlinear Soft Matter Phys. 76 (December (6 Pt 1)), 061304.

Antony, S.J., Kuhn, M.R., 2004. Influence of particle shape on granular contact signatures and shear strength: new insights from simulations. Int. J. Solids Struct. 41 (21), 5863-5870.

Azéma, E., Radjai, F., 2010. Stress-strain behavior and geometrical properties of packings of elongated particles. Phys. Rev. E 81 (5 (Part 1 )), 051304.

Azéma, E., Saussine, G., Radjai, F., 2009. Quasistatic rheology, force transmission and fabric properties of a packing of irregular polyhedral particles. Mech. Mater. 41 (6), 729-741.

Bardenhagen, S., Brackbill, J., Sulsky, D., 2000a. The material-point method for granular materials. Comput. Methods Appl. Mech. Eng. 187, 529-541.

Bardenhagen, S., Guilkey, J., Roessig, K., Brackbill, J., Witzel, W., Foster, J., 2001. An improved contact algorithm for the material point method and application to stress propagation in granular material. Comput. Model. Eng. Sci. 2, 509-522.

Bardenhagen, S.G., Brackbill, J.U., Sulsky, D., 2000b. Numerical study of stress distribution in sheared granular material in two dimensions. Phys. Rev. E 62, $3882-3890$

Berryman, J.G., 1986. Random close packing of hard spheres and disks. Phys. Rev. A 27, 1053.

Bonnecaze, R., Cloitre, M., 2010. Micromechanics of soft particle glasses. Adv. Polym. Sci. 236, 117-161.

Brogliato, B., 1999. Nonsmooth Mechanics. Springer, London.

CEGEO, Saint-Cyr, B., Szarf, K., Voivret, C., Azéma, E., Richefeu, V., Delenne, J.-Y., Combe, G., Nouguier-Lehon, C., Villard, P., Sornay, P., Chaze, M., Radjai, F.,

2012. Particle shape dependence in 2d granular media. EuroPhys. Lett. 98, 44008.

Cloitre, M., Borrega, R., Monti, F., Leibler, L., 2003. Glassy dynamics and flow properties of soft colloidal pastes. Phys. Rev. Lett. 90, 068303.

Cruz, F.D., Chevoir, F., Bonn, D., Coussot, P., 2002. Viscosity bifurcation in granular materials, foams, and emulsions. Phys. Rev. E 66 (5 (Pt 1)), 051305.

Cummins, S., Brackbill, J., 2002. An implicit particle-in-cell method for granular materials. J. Comput. Phys. 180, 506-548.

Cundall, P.A., Strack, O.D.L., 1979. A discrete numerical model for granular assemblies. Géotechnique 29 (1), 47-65.

Donev, A., Cisse, I., Sachs, D., Variano, E.A., Stillinger, F.H., Connelly, R., Torquato, S., Chaikin, P.M., 2004. Improving the density of jammed disordered packings using ellipsoids. Science 303 (5660), 990-993.

Donev, A., Connelly, R., Stillinger, F., Torquato, S., 2007. Underconstrained jammed packings of nonspherical hard particles: ellipses and ellipsoids. Phys. Rev. E 75, 051304.

Gatta, J.-M., Moneriea, Y., Lauxa, D., Baronb, D., 2005. Elastic behavior of porous ceramics: application to nuclear fuel materials. J. Nucl. Mater. 336, $145-155$. GDR-MiDi, 2004. On dense granular flows. Eur. Phys. J. E 14, 341-365.

Guilkey, J., Weiss, J., 2003. Implicit time integration for the material point method: quantitative and algorithmic comparisons with the finite element method. Int. J. Numer. Methods Eng. 57, 1323-1338.

Herrmann, H.J., Mahmoodi Baram, R., Wackenhut, M., 2003. Searching for the perfect packing. Physica A: Stat. Mech. Appl. 330 (December (1-2)), 77-82.

$\mathrm{Hu}$, W., Chen, Z., 2003. A multi-mesh mpm for simulating the meshing process of spur gears. Comput. Struct. 81, 1991-2002.

Huang, P., Zhang, X., Ma, S., Huang, X., 2011. Contact algorithms for the material point method in impact and penetration simulation. Int. J. Numer. Methods Eng. 85, 498-517.

Jaeger, H., Nagel, S., 1996. Granular solids, liquids and gases. Rev. Mod. Phys. 68, 1259-1273.

Jean, M., 1995. Frictional contact in rigid or deformable bodies: numerical simulation of geomaterials. In: Salvadurai, A., Boulon, J. (Eds.), Mechanics of Geomaterial Interfaces. Elsevier Science Publisher, Amsterdam, pp. 463-486.

Jean, M., 1998. The non-smooth contact dynamics method. Comput. Methods Appl. Mech. Eng. 177, $235-257$.

Johnson, K., 1999. Contact Mechanics. University Press, Cambridge.

Kruyt, N.P., 2003. Contact forces in anisotropic frictional granular materials. Int. J. Solids Struct. 40 (13-14), 3537-3556

Leroy, B., 1985. Collision between two balls accompanied by deformation: a qualitative approach to Hertz's theory. Am. J. Phys. 53 (4), $346-349$.

Majmudar, T.S., Behringer, R.P., 2005. Contact force measurements and stress-induced anisotropy in granular materials. Nature 435 (June (7045)), 1079-1082.

Matuttis, H.-G., Luding, S., Herrmann, H.J., 2000. Discrete element methods for the simulation of dense packings and heaps made of spherical and nonspherical particles. Powder Tech. 109, 278-292.

Moreau, J., 1994. Some numerical methods in multibody dynamics: application to granular materials. Eur. J. Mech. A/Solidso 13 (4), $93-114$.

Moreau, J.J., 1988. Bounded variation in time. In: Panagiotopoulos, P., Strang, G. (Eds.), Topics in Nonsmooth Mechanics. Bikhäuser, Basel, pp. 1-74.

Nedderman, R.M., 1992. Statics and Kinematics of Granular Materials. Cambridge University Press, Cambridge.

Pan, X., Xu, A., Zhang, G., Zhang, P., Zhu, J., Ma, S., Zhang, X., 2008. Three-dimensional multi-mesh material point method for solving collision problems. Commun. Theor. Phys. 49, 1129-1138.

Rabinowicz, E., 1965. Friction and Wear of Materials. Wiley, New York. 
Radjai, F., Dubois, F., 2011. Discrete Numerical Modeling of Granular Materials. Wiley-ISTE, New York.

Radjai, F., Jean, M., Moreau, J., Roux, S., 1996. Force distributions in dense two-dimensional granular systems. Phys. Rev. Lett. 77, 274.

Radjai, F., Richefeu, V., 2009. Contact dynamics as a nonsmooth discrete element method. Mech. Mater. 41, 6715-6728.

Radjai, F., Troadec, H., Roux, S., 2004. Key features of granular plasticity. In: Antony, S., Hoyle, W., Ding, Y. (Eds.), Granular Materials. RSC, Cambridge, pp. 157-184.

Radjai, F., Wolf, D.E., Jean, M., Moreau, J., 1998. Bimodal character of stress transmission in granular packings. Phys. Rev. Lett. 80, 61-64.

Richefeu, V., 2009. El Youssoufi, 2009. Force transmission in dry and wet granular media. Powder Tech. 190, $258-263$.

Roberts, A.P., Teubner, M., 1995. Transport properties of heterogeneous materials derived from gaussian random fields: bounds and simulation. Phys. Rev.

51, 4141.

Saint-Cyr, B., Delenne, J.-Y., Voivret, C., Radjai, F., Sornay, P., 2011. Rheology of granular materials composed of nonconvex particles. Phys. Rev. E 84 (4), 041302.

Torquato, S., Truskett, T.M., Debenedetti, P.G., 2000. Is random close packing of spheres well defined? Phys. Rev. Lett. 84 (March (10)), 2064.

Turner, A., Schuster, R., 1996. Landslides: Investigation and Mitigation. Transp. Res. Board, Nat. Res. Council, Washington.

Unger, T., Brendel, L., Wolf, D.E., Kertész, J., 2002. Elastic behavior in contact dynamics of rigid particles. Phys. Rev. E: Stat. Nonlinear Soft Matter Phys. 65 $(6$

(Pt 1)), 061305.

Voivret, C., Radjai, F., Delenne, J.-Y., Youssoufi, M.S.E., 2007. Space-filling properties of polydisperse granular media. Phys. Rev. E 76 (2 (Pt 1)), 021301.

Wood, D., 1990. Soil Behaviour and Critical State Soil Mechanics. Cambridge University Press, Cambridge, England. 